ISSN 1991- 8690

website : http:// jsci.utq.edu.iq
الترقيم الدولي • 1991 - 199

Email: utjsci@utq.edu.íq

\title{
Noise Characteristics in Presence of Four Wave Mixing in Single Mode Fibers
}

\author{
Haider K. Muhammad ${ }^{(1)}$
}

Ali H. Hassan

(2)

Hassan A. Yasser

(1)

\author{
${ }^{(1)}$ ThiQar University- Science College
}

$\underline{\text { Abstract }}$

(2) Al-Mustansyria University-Education College

The transmission system in the fiber optics affected by many conventional noises. In case of employing WDM systems which means the addition of further new noises named nonlinear noises. As far As the FWM is the main important phenomena noise, all sources of noises being analyzed and a new equation has been set for the FWM .Accordingly the total influence on the allowable input power has been determined, then a general form equation connecting between allowable power and Q-factor has been derived. Since FWM depends on the phase-matching a comprehensive equation has been derived depending on the center of the process mixing.

\section{تحليلات الضوضاء بوجود ظاهرة مزج الموجات الاريع في الالياف احادية النمط

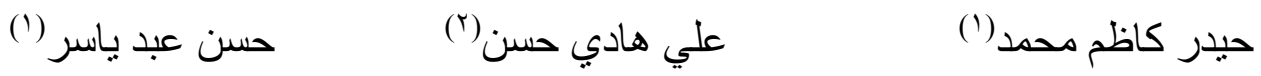

الخلاصة

يتأثر نظام الإرسال في الألياف البصرية بعدد من مصادر الضوضاء الثقليدية. في حالة استخدام أنظمة مزج-قسمة التزدد فهذا يعني أرسال أكثر من

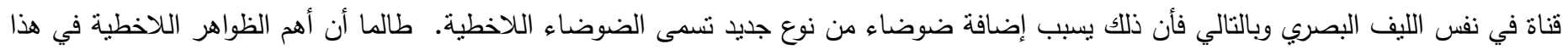

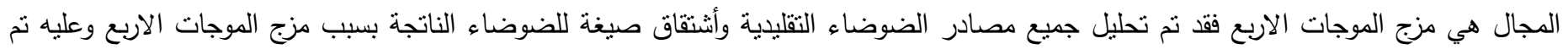

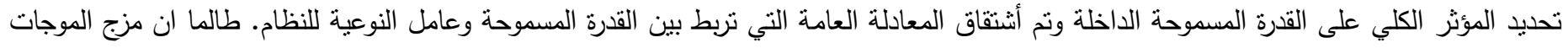
الاربع يعتد بقوة على شرط تطابق الطور فقد بينا صيغة مطورة لثرط تطابق الطور تعتمد في الاساس على مركز عملية مزج الموجات الاربع.

\section{Introduction}

In wavelength division multiplexing (WDM) systems, four wave mixing (FWM) is the most influential effect [1]. When FWM occurs, three input signals generate a fourth signal, called FWM signal, which may affect the input signal operating at the same wavelength [2]. Obviously, the performance of multiwavelength WDM system may be seriously affected by FWM crosstalk due to the interaction of various combinations of the active signal wavelengths [3]. WDM system greatly increase the total bandwidth of each optical fiber using a number of closely spaced channels at wavelengths within the typical $1540 \mathrm{~nm}$ to $1560 \mathrm{~nm}$ to take advantage of the "low loss" transmission window in optical fibers and to enable the use of erbium doped fiber amplifiers EDFA's [1,3]. Any interaction between these channels will lead to degradation of the bit error rate (BER) of the system for two reasons. Firstly, the pump channels will experience signal depletion as optical power is transferred to a different wavelengths. Secondly, if the frequency of a FWM product coincides with one of the allocated system channels, then this channel will suffer from noise. This is a particular problem for channels that are equally spaced in frequency $[3,4,5]$. It should be noted that each optical channel is completely independent of the other optical channels. It may run at its own rate (speed) and use its own encodings and protocols without any dependence on the other channels at all $[5,6]$. Channels speed for wide area network (WAN) applications are typically 2.4 Gbps in current 
operational WDM systems [3]. One way of minimizing the impact of FWM is to place WDM channels such that the generated signals do not fall within other WDM channels. Thus they don't interfere with other channels too much. This does help but it cannot overcome the problem of noise generated in the source WDM channels by power being transferred out of them. In addition, some WDM devices are difficult to construct if wavelength spacing is uneven (reflective gratings for example) $[5,6]$. In this paper, the different types of conventional noises in optical fiber system are analyzed. Also, the nonlinear noise due to FWM is derived. As a consequence, the total effected noise is determined, which may be used to control the allowable input power that will be used in different WDM system. However, the FWM is controlled by the phase matching condition that was computed by an adopted expression.

\section{Conventional Noise Sources}

The goal of the photo receiver is to sample the signal at the decision time and to make decision concerning its value. The decision circuit compares the received sampled value to the threshold value $I_{\text {th }}$ and forces the bit value to ' 1 ' when $I_{i h}<I$ and to ' 0 ' when $I_{t h}>I$. An error occurs when $I_{t h}<I$ whereas the value of the bit symbol is ' 0 ' and vice versa. Under the assumption that the distribution of the different sources of noise is Gaussian, one can be deduced that the distribution of noise on symbols ' 0 ' and '1' is also Gaussian and can be defined by their variances $\sigma_{1}^{2}$ and $\sigma_{0}^{2}$. Note that, all analysis in this section deals with the ON-OFF keying (OOK) format, where each signal represents one bit only. For other format modulation systems, the analysis must be modified. The bit error ratio under the assumption $\left(I_{1}-I_{h h}\right) / \sigma_{1}=\left(I_{t h}-I_{0}\right) / \sigma_{0}$ can be expressed as $[7,8]$

$$
B E R=\frac{1}{\sqrt{\pi}} \int_{e}^{\infty} e^{-y^{2}} d y
$$

where $Q=\left(I_{1}-I_{0}\right) /\left(\sigma_{1}+\sigma_{0}\right)$ represents the quality factor. There are different types of noise that may be introduced at the received pulses. In the case of no noise at the input, they are: shot noise, thermal noise, beating noise, and the nonlinear noise. In the following subsections, we will abbreviate the necessary mathematical expressions of these types in order to obtain an accurate relation between the allowable signal power and the quality factor in WDM systems. The beating noise is due to the interference between optical signals at the photodiode input, which are signalamplified spontaneous emission (ASE) noise and ASEASE noise. These noises are defined as [8,9]

$$
\begin{aligned}
& \sigma_{s-A S E}^{2}=4(h v) G^{2} K^{2} B_{e} P_{s} \\
& \sigma_{A S E-A S E}^{2}=(h v)^{2} M G^{2} K^{2}\left(2 B_{o}-B_{\varepsilon}\right) n_{e q}^{2}
\end{aligned}
$$

where $K=\eta e / h v$ represents the responsivity that is a measure of photodiodes sensitivity, $P_{s}$ is the input power, ${ }^{B_{o}}$ is the optical bandwidth of the amplifier, $B_{e}$ is the bit rate, $h v$ is the photon energy, $G$ is the amplifier gain, $n_{e q}$ is the equivalent input noise factor that matches the spontaneous emission factor, ${ }_{s p}$, at high gain as $n_{e q}=n_{s p}(G-1) / G$, and $M$ is the number of ASE modes. Shot noise raises from the statistical nature of the generation and collection of photoelectrons when an optical signal is incident on a photodiode, which is related to quantum characteristics of photocurrent. Its expression is given by [10]

$\sigma_{\text {shot }}^{2}=2 \eta e B_{s}\left(G I_{s s}+M I_{N}\right)$

where $\eta$ is the coupling efficiency, $e$ represents the electron charge, $I_{s}=P_{s} e / h v$ is the photocurrent due to optical signal, $P_{s}$ is the input power at the receiver, $I_{N}=P_{A S E} e / h v$ is the photocurrent due to ASE noise corresponding to the noise power $P_{A S E}$. The shot noise at the bit ' 0 ' is due to ASE only, while the noise at the bit ' 1 'are related to ASE and signal power. Mathematically, the shot noises will be

$$
\begin{aligned}
\sigma_{\text {thor } 0}^{2} & =2(h v)^{2} M G K B_{s} B_{0} n_{e q} \\
\sigma_{\text {thort }}^{2} & =2(h v) M G K B_{s} B_{0} n_{e q}+2 e G K B_{s} B_{0} P_{x}
\end{aligned}
$$

where $K=\eta e / h v$ represents the responsivity that is a measure of photodiodes sensitivity. Thermal noise originates within the photodiode resistance $R$. Electrons within any resistor never remain stationary. They continuously move because of their thermal energy even with no applied voltage. The electron motion is random, so the net flow of charge could be towards one electrode or the other at any instant. Thus, the mean square value of this current is given by [11]

$\sigma_{\dot{*}}^{2}=\frac{4 B_{\varepsilon} k_{s} T}{R}$ 
where ${ }^{k_{B}}$ is Boltzmann's constant and $T$ is the absolute temperature.

\section{Four Wave Mixing}

For a WDM system with $\mathrm{N}$ channels, the number of products $\mathrm{M}$ will be $M=\left(N^{3}-N^{2}\right) / 2$ [8]. Clearly, for a typical 8-channels WDM system, unless great care is taken to allocate the channel frequencies carefully, some crosstalk will occur due to FWM. With 16 and 32-channels WDM systems on the horizon, FWM is a serious consideration in a system design. Crosstalk can be defined as a small proportion of the optical power that should have ended up in a particular channel actually ends up in an adjacent (or another) channel $[1,2]$. Crosstalk is critically important in WDM systems, when signals from one channel arrive in another they become noise in the other channel. This can have serious effects on the signal to noise ratio (SNR) and hence on the error rate of the system $[3,6]$.

The FWM is a third order nonlinear effect in which three optical waves at frequencies $f_{n}, f_{m}$, and $f_{k}$ mix to originate a new wave at frequency

$$
f_{\text {wnx }}=f_{n}+f_{m}-f_{k}
$$

Considering that the input continuous waves are not depleted by the generation of mixing products, and that the states of polarization of these waves are coincided and not changing along the propagation, the optical power of the new generated wave at frequency $f_{n m k}$ is given by [12]

$$
P_{n n k \dot{k}}=d^{2} P_{n} P_{m} P_{k} b \eta_{m n k}
$$

Where $\eta_{n m k}$ is the FWM efficiency and $d$ is the degeneracy factor, which takes value 1 and 2 for degenerate and non-degenerate terms, respectively, and

$$
\begin{aligned}
& b=\gamma^{2} e^{-\alpha L}\left(1-e^{-\alpha L}\right)^{2} / \alpha^{2} \\
& \eta_{\mathrm{nmk}}=\frac{\alpha^{2}}{\alpha^{2}+\Delta \beta_{n m k}^{2}}
\end{aligned}
$$

where $\gamma$ is the nonlinearity coefficient, $\alpha$ is the fiber attenuation, $\mathrm{L}$ is the fiber length, and $P_{n}, P_{m}, P_{k}$ are the input power of channels $n, m$, and $k$, and $\Delta \beta_{n m k}=\beta_{n}+\beta_{m}-\beta_{k}$ is phase mismatch.
If the mixing center is $\omega_{c}=\left(\omega_{n}+\omega_{m}\right) / 2$ the $\Delta \beta_{n m k}$ determined by using Taylor expansion about $\omega_{c}$. Consequently $\beta_{n}, \beta_{m}, \beta_{k}$, and $\beta_{l}$ are expanded about $\omega_{c}$ to get [13]

$$
\Delta \beta_{i k}=\sum_{K=1}^{4} \frac{\beta_{K}}{K !}\left[\left(\omega_{i}-\omega_{c}\right)^{2 K}+\left(\omega_{j}-\omega_{c}\right)^{2 x}-\left(\omega_{k}-\omega_{c}\right)^{2 x}\left(\omega_{i}-\omega_{c}\right)^{2 x}\right]
$$

where the orders of $\beta_{K}$ in case of $K \geq 5$ are discarded. Processing Eq.(10) will illustrate that the odd terms are zeros. That is; the residual terms are the second and forth orders. The second order dispersion is defined as [1]

$\beta_{2}\left(\omega_{c}\right) \approx \beta_{30}\left(\omega_{c}-\omega_{\circ}\right)+\frac{\beta_{40}}{2}\left(\omega_{c}-\omega_{\mathrm{o}}\right)^{2}$

where $\omega_{0}=2 \pi c / \lambda_{0}$ and the parameters $\beta_{30}, \beta_{40}$ for silica are assumed to be constant with typical values $\beta_{30}=0.1 \mathrm{ps}^{3} / \mathrm{km}$ and $\beta_{40}=10^{-4} \mathrm{ps}^{4} / \mathrm{km}$ [14]. One can notice from Eq.(11), $\beta_{2}$ is possible to be negative or positive depending on the location of FWM process center with respect to $\lambda_{\circ}$. By using the definition $\omega_{l}=\omega_{n}+\omega_{m}-\omega_{k}$ into Eq.(10), it is easy to conclude the following phase matching condition

$$
\Delta \beta_{\text {nonk }}=-\left\{\beta_{2}\left[\left(\omega_{c}-\omega_{k}\right)^{2}-\omega_{d}^{2}\right]+\frac{\beta_{40}}{12}\left[\left(\omega_{c}-\omega_{k}\right)^{4}-\omega_{d}^{4}\right]\right\}
$$

where $\Delta \beta_{n m k}$ for each $(n, m, k)$ FWM process will be computed depending on $\omega_{d}=\left(\omega_{n}-\omega_{m}\right) / 2$, $\omega_{c}=\left(\omega_{n}+\omega_{m}\right) / 2, \omega_{0}$, and using Eqs.(11) and (12).

This paper considers a WDM system with $\mathrm{N}$ equally spaced wavelengths. At a particular wavelength frequency $f_{l}$, the FWM waves generate from various combinations of interacting signals. The total FWM power at $f_{m}$ is given by

$$
P_{\text {tot }}\left(f_{i}\right)=\sum_{f_{n}, f_{m}, f_{k}=f_{n}+f_{m}-f_{i}} P_{R W}\left(f_{n}, f_{m}, f_{k}\right)
$$

where $n, m, k=1,2,3, \ldots \ldots \ldots ., N, n \neq l, m \neq l$ and the number $l$ takes the values: $-(N-1), . .,-1,0,1, \ldots \ldots \ldots \ldots . . . . . .2 N-1$ which represent the indices of the new frequencies generated. 
The power generated by a signal with $f_{l}$ frequency, Eq.(13), is resulted from the fact that all the channels with the frequencies $f_{n}, f_{m}$, and $f_{k}$ are in ON state. But there is a possibility for any channel to be in OFF state, thus Eq.(13) can be expressed as

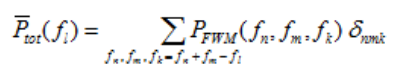

where $\delta_{n m k}=m_{n} * m_{m} * m_{k}$ and $m_{n}, m_{m}, m_{k}$ represent the modulation factors in the $n, m, k$ wavelength, respectively.

where

$$
\delta_{n m k}=\left\{\begin{array}{lr}
1 & \text { if } m_{\mathrm{n}}=m_{\mathrm{m}}=m_{\mathrm{k}}=1 \\
0 & \text { elsewhere }
\end{array} .\right.
$$

\section{Nonlinear Noise}

There are different types of nonlinear noises, such as SPM noise, XPM noise, FWM noise...etc. In this paper, the important effect is FWM, so the nonlinear noise is represented by the FWM noise that is formed as [10]

$\sigma_{F W M}^{2}=N^{(m)} G^{2} K^{2} P_{s}$

where

$$
N^{(m)}=2\left[\frac{1}{8} \sum_{\mathrm{NFWM}} P_{i j k}+\frac{1}{4} \sum_{\mathrm{DFWM}} P_{i i k}\right]
$$

Note that, the parameters $\sum_{\text {NFWM }} P_{i j k}$ and $\sum_{D F W M} P_{i k}$ represent the sum of the generated powers at the frequency $f_{m}$ due to NFWM and DFWM, respectively. The factors $1 / 8$ and $1 / 4$ in last equation are attributed to the following scenario: the NFWM take places if the three bits are ' 1 ', which happens at a probability $1 / 2^{3}=1 / 8$, while the DFWM take places if two bits are '1', which happens with a probability $1 / 2^{2}=1 / 4$. Now, assuming that the input channels have the same power $P_{0}$, such that the parameter $c^{(m)}$ is reformed as

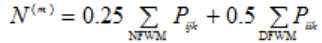

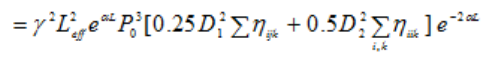

where $D_{1}$ and $D_{2}$ represent the degeneracy factors of NFWM and DFWM, respectively.

\section{Allowable Input Power}

The total variances of currents are calculated as

$$
\begin{aligned}
& \sigma_{0}^{2}=\sigma_{\text {sker } 0}^{2}+\sigma_{\text {ASE- MSE }}^{2}+\sigma_{s k}^{2}+\sigma_{F W M}^{2}
\end{aligned}
$$

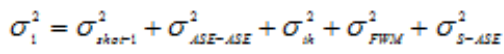

Substituting Eqs.(2) to (5) and (15) into (18) and using the fact $P_{s}=e^{-\alpha L} P_{0}$, yields

$$
\begin{aligned}
\sigma_{0}^{2} & =K^{2} G^{2}\left[a+N^{(\pi)} P_{0} e^{-a t}\right] \\
\sigma_{1}^{2} & =K^{2} G^{2}\left[a+b P_{0} e^{-a t}+N^{(m)} P_{0}^{4} e^{-4 a t}\right]
\end{aligned}
$$

\section{Where}

$$
a=B_{\varepsilon}^{2}(h v)^{2}\left[\frac{2 B_{o} M n_{e q}}{B_{\varepsilon} \eta G}+\frac{M n_{\varepsilon q}^{2}\left(2 B_{o}-B_{\varepsilon}\right)}{B_{\varepsilon}}+\frac{4 k_{B} T}{B_{\varepsilon} e^{2} \eta^{2} G^{2} R}\right], b=B_{\varepsilon}(h v)\left[\frac{2}{\eta G}+4 n_{\varepsilon q}\right]
$$

The current difference is computed as

$$
I_{1}-I_{0}=\eta\left(G I_{s}+M I_{N}\right)-\eta M I_{N}=K G P_{s}
$$

Substituting Eqs.(19) and (20) into $Q=\left(I_{1}-I_{0}\right) /\left(\sigma_{1}+\sigma_{0}\right)$, we get

$Q=\frac{P_{0} e^{-a L}}{\sqrt{a+N^{(\pi)} P_{0}^{4} e^{-4 \alpha L}}+\sqrt{a+b P_{0} e^{-a L}+N^{(\pi)} P_{0}^{4} e^{-4 \alpha L}}}$

Squaring and rearranging of Eq.(21) two times, will obtain

$$
4 Q^{2} N^{(m)} P_{0}^{4}-P_{0}^{2} e^{2 a r}+2 b Q^{2} P_{0} e^{3 a x}+\left(b^{2} Q^{4}-4 a Q^{2}\right) e^{4 a t}=0
$$

Eq.(22) represents the input power to the receiver taking into accounts the effects of FWM. It will be solved numerically to determine the allowable input power in the WDM system. This equation is considered as an important contribution in this paper. To our best knowledge, there are no published studies related to noise analysis that address these effects simultaneously. The simplest cases have been studied in many scientific researchers using different approaches [14-16]. Note that, with FWM only, we have $a=b=0$. As a consequence, Eq.(22) will be

$$
P_{0}=\frac{e^{-o L}}{2 \sqrt{N^{(m)}} Q}
$$

while the absence of FWM will make $P_{0}=e^{-a L}\left(b Q^{2}+\sqrt{a} Q\right)$ 
Note that, the presence of FWM only will make the allowable power is inversely changed with the desired quality factor, while the absence of FWM will make the allowable power is a quadratic function of quality factor. For higher bit rates, the parameters $a, b$ will become comparable to $N^{(m)}$, such that the allowable input power will be restricted by all types of noise.

\section{Simulation Results and Discussion}

Our simulation of the transmission is characterized by a large number of different parameters, most of which do not change from simulation to simulation. for completeness, we list a typical parameter set below, and maintain deviation from these values whenever they occur [13]

$$
\begin{array}{lllll}
\beta_{3}=0.1 \mathrm{ps}^{3} / \mathrm{Km} & \beta_{4}=1^{*} 10^{-4} \mathrm{ps}^{4} / \mathrm{Km} & \mathrm{T}=300 \mathrm{k}^{\circ} & L=20 \mathrm{Km} & \mathrm{B}_{\mathrm{g}}=70 \mathrm{GHs} \\
P_{\mathrm{g}}=0.001 \mathrm{mw} & \alpha=0.2 \mathrm{~dB} / \mathrm{km} & \lambda_{\mathrm{g}}=1550 \mathrm{~mm} & \mathrm{M}=2 & \mathrm{n}_{\mathrm{q}}=2 \\
\mathrm{e}=1.6 \mathrm{e}-19 \mathrm{C} & \gamma=4 \mathrm{~W}^{4} \mathrm{Km}^{4} & \mathrm{k}_{\mathrm{g}}=1.38 \mathrm{e}^{-23 \mathrm{~J} / \mathrm{K}} & \mathrm{R}=500 \Omega & \mathrm{G}=2
\end{array}
$$

It is desirable that the power of the signal will not be larger than the above value to avoid stimulated Raman scattering (SRS). Fig.(1) shows the quality factor as a function of allowable input power for different WDM systems, where the continuous and dotted lines represented the present method also the discrete and dotted-discrete lines represented the conventional method with all noises and FWM only respectively. It is clear that the middle channels is more effected that the first channel in all conditions, on the other side the increase of the channels of the system will decrease of achieve quality facer Q.The performed Q of the first channel is larger than that of middle channels, relying on the same work conditions for all $\mathrm{N}$ values. Form our method of calculation it is clear that all cases give us lager $Q$ value, this because the present phase matching give us less value of FWM generated. From the facts mentioned above we conclude that the system is facing less distortion by FWM. Fig.(2) illustrates The FWM components number as a function of channel index for different WDM system. It is obvious from this Fig. the increase of the number of channels lead to considerable increasing in the number of the generated components, and the majority of this increase is situated in the middle channels of the system. This rise in the number of components doesn't mean an increase in the power every channel. since the generated power depending on the efficiency mixing which rely on the phase matching. Finally all cases in Fig.(1) have proved the addition of traditional noises (as in Fig.(3)) changing the value of
Q in about $(-10 \mathrm{db})$ depending on the quantity of achieved bit rate and the other parameters of the system, i.e. the increase of bit rate lead to decrease of performed $\mathrm{Q}$. The input power required to achieve the same $Q$ value will increase with $\mathrm{L}$, and the reason why, it is essential to overcome the effect of the noises which in turn increase with the L.

\section{Conclusions}

An analysis is presented to evaluate the limitations imposed by conventional noises and nonlinear noise (FWM only) on allowable input power in optical WDM systems. Regarding the combination of all types of above mentioned noises coincide with the results obtained by our assumption which is in accordance with real physical behavior. Also a better values of BER when a new phase-matching factor was introduced, which depends on the center of the process mixing not on the center of the system.
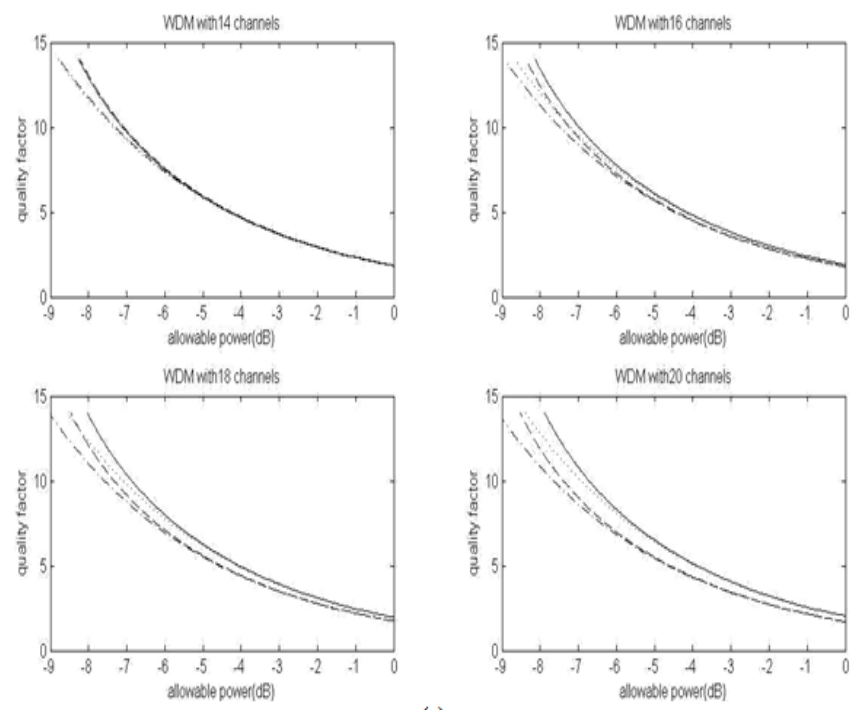

(a) 

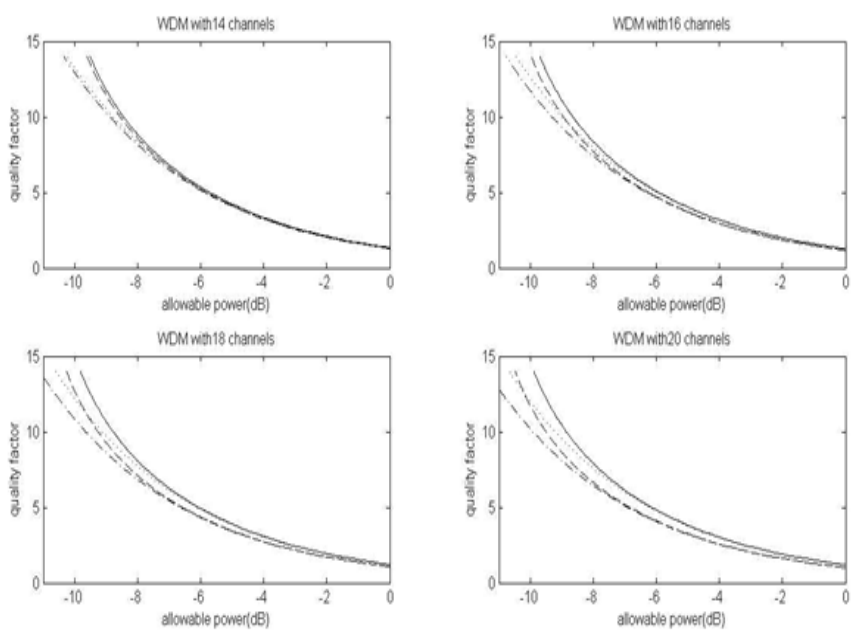

(b)

Fig.(1): the quality factor as a function of allowable input power for different WDM systems, where the continuous and dotted lines represent the present method, while the discrete and dotted-discrete lines represent the conventional method with all noises and FWM only respectively: a) the behavior of first channel, b) the behavior of central channel.
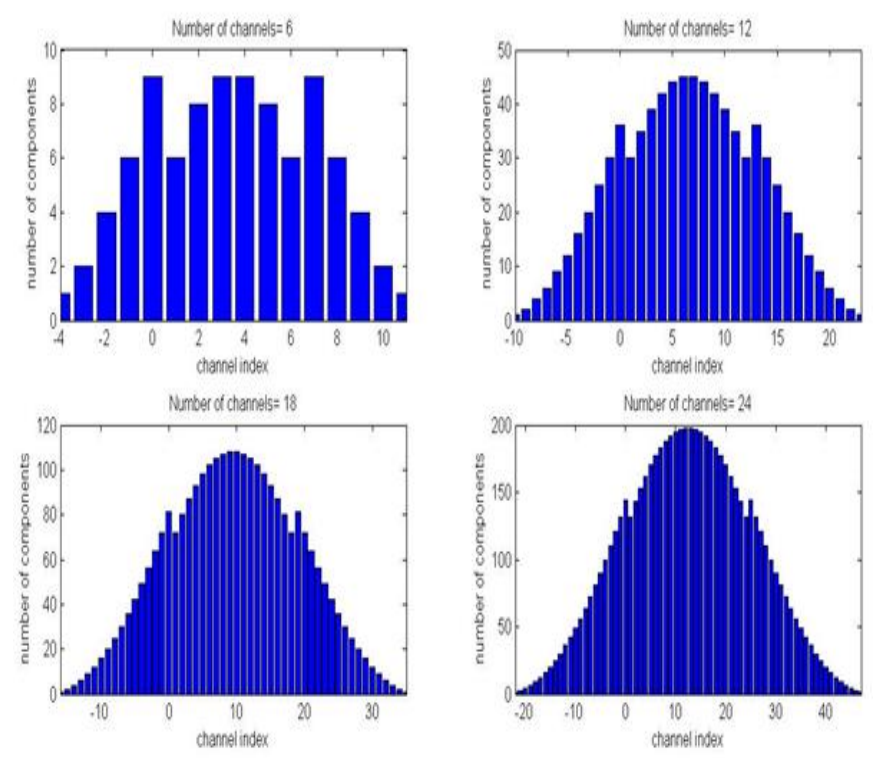

Fig.(2): The FWM components number as a function of channel index for different WDM system.
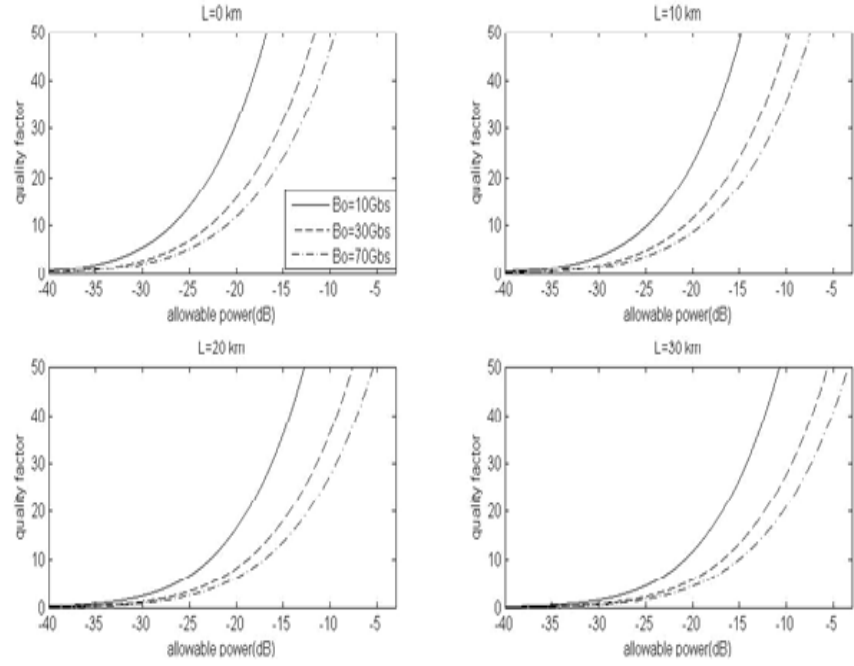

Fig.(3): The quality factor as a function of allowable input power for different lengths, where the continuous, discrete, and dotted-discrete lines represents the bit rates 10,30 , and $70 \mathrm{GBs}$ respectively.

\section{References}

[1] G. Agrawal, "Nonlinear Fiber Optics", 4th Edition, Academic Press, 2007.

[2] J. Palais, "Fiber Optic Communications", 4th Edition, Prentice Hall, 1998.

[3] S. Mechels, J. Schlger, and D. Franzen, "Accurate Measurements of the Zero Dispersion Wavelength in Optical Fibers", J. Res. Natl. Inst. Stand. Tec., Vol.102, No.3, pp.333-347, 1997.

[4] J. Heebner, "Nonlinear Optical Whispering Gallery Micro-resonators for Photonics", Ph. D. Thesis, Rochester University, 2003.

[5] B. Kuhlmey, R. McPhedran, P. Robinson, G. Renversez, and D. Maystre, "Microstructured Optical Fibers: Where's the Edge?", Optics Express, Vol.10, No.22, pp.1285-1290, 2002.

[6] U. Osterberg, "Signal Processing in Optical Fibers", Modern Signal Processing MSRI, Vol.46, pp.301-316, 2003.

[7] E. Mutafungwa and S. Halme, "On the Use of Optical Spreading Codes to Design DWDM 
Systems with Reduced FWM Crosstalk”, Optics Communications 198, pp. 339-350, 2001.

[8] I.Neokosmidis,et.al "New Techniques for the Suppression of the Four-Wave Mixing-Induced Distortion in Nonzero Dispersion Fiber WDM Systems", J. OF Lightwave Tec., VOL. 23, NO. 3, MAR. 2005.

[9] K. Inoue, H. Toba, and K. Oda, "Influence of Fiber Four- Wave Mixing on Multichannel FSK Direct Detection Transmission Systems", J. of Lightwave Tec., vol. 10, No. 3, March 1992.

[10] Y. Nagatani at el. "Theoretical Analysis of Frequency Allocations in FDM Lightwave Transmission Systems" J. Lightwave Tec., Vol. 26, No. 13, July 1, 2008.

[11] K. Inoue and H. Toba, "Fiber Four-Wave Mixing in Multi- Amplifier Systems with Nonuniform Chromatic Dispersion", J. of Lightwave Tec., vol. 13, No. 1, January 1995.

[12] K. Hill, D. Johnson, B.S. Kawasaki, and R. I. MacDonald, "CW three-wave mixing in singlemode optical fibers,” j. Appl. Phyx, vol. 49, pp. 5090-5106, 1978.

[13] H. Muhammad, A. Hassan and H. Yasser "Four Wave Mixing in Equally Spaced Wavelength Division Multiplexing system", the 20th scientific Conference of College Education, ALMustansirya University, 24- 25 April, 2013. under publishing.

[14] A.Yriv and P.Yeh "Optical Electronics in Modern Communications", Oxford University press, 2007.

[15] O. Aso, M. Tadakuma, and S. Namiki, "Four Wave Mixing in Optical Fibers and its Applications", Furukawa Review, No.19, pp.6368, 2000.

[16] M. Marhic, K. Wang, M. Ho, and L. Kazovsky, "92\% Pump Depletion in Continuous Wave One Pump Fiber Optical Parametric Amplifier", Optics. Let., Vol.26, No.9, pp.620-622, 2001. 